\title{
GMDH-based Models for Mid-term Forecast of Cryptocurrencies (on example of Waves)
}

\author{
Pavel Mogilev \\ Technological University Dublin, nismorus@gmail.com \\ Anna Boldyreva \\ Moscow Institute of Physics and Technology, anna.boldyreva@phystech.edu \\ Mikhail Alexandrov \\ Autonomous University of Barcelona, malexandrov@mail.ru
}

See next page for additional authors

Follow this and additional works at: https://arrow.tudublin.ie/ittscicon

Part of the Computer Sciences Commons

\section{Recommended Citation}

P. Mogilev, A. Boldyreva, M. Alexandrov and J. Cardiff, "GMDH-based Models for Mid-term Forecast of Cryptocurrencies (on example of Waves)," 2020 IEEE 15th International Conference on Computer Sciences and Information Technologies (CSIT), 2020, pp. 13-16, doi: 10.1109/CSIT49958.2020.9321873.

This Conference Paper is brought to you for free and open access by the School of Science and Computing at ARROW@TU Dublin. It has been accepted for inclusion in Conference Papers by an authorized administrator of ARROW@TU Dublin. For more information, please contact arrow.admin@tudublin.ie, aisling.coyne@tudublin.ie,gerard.connolly@tudublin.ie. 


\section{Authors}

Pavel Mogilev, Anna Boldyreva, Mikhail Alexandrov, and John Cardiff 


\section{GMDH-based Models for Mid-term Forecast of Cryptocurrencies (on example of Waves)}

\author{
Pavel Mogilev \\ Technological University of Dublin \\ Dublin, Ireland \\ nismorus@gmail.com \\ Mikhail Alexandrov \\ RANEPA \\ Moscow, Russia \\ Autonomous University of Barcelona \\ Barcelona, Spain \\ malexandrov@mail.ru
}

\author{
Anna Boldyreva \\ Moscow Institute of Physics and Technology \\ Moscow, Russia \\ anna.boldyreva@phystech.edu \\ John Cardiff \\ Technological University of Dublin \\ Dublin, Ireland \\ john.cardiff@tudublin.ie
}

\begin{abstract}
Cryptocurrencies became one of the main trends in modern economy. However by the moment the forecast of cryptocurrencies values is an open problem, which is almost non-reflected in publications related to finance market. Reasons consist in its novelty, large volatility and its strong dependence on subjective factors. In this experimental research we show possibilities of GMDH-technology to give weekly and monthly forecast for values of cryptocurrency 'Waves' (waves/euro rate). The source information is week data covering the period 2017-2019. We tests 4 algorithms from the GMDH Shell platform on the whole period and on the crisis period 4-th quarter 2017 - 2nd quarter 2018. Baseline is provided by the popular statistical method of double exponential smoothing. The results of Pilot study can be considered as the very promising ones having in view the large variability of data.
\end{abstract} Shell

Keywords-cryptocurrency, time series, GMDH, GMDH

\section{INTRODUCTION}

\section{A. Motivation}

In this paper we use the following principal terms:

- Blockchain. It is a continuous sequential chain of blocks containing information about transactions. Blockchain of bitcoins is a public record of all bitcoin transactions.

- Cryptocurrency. It is a type of digital currency, whose creation and control is based on cryptographic methods. As a rule, cryptocurrency accounting is decentralized.

- ICO. It is an attraction of investments in the form of selling to investors a fixed number of new units of cryptocurrencies received by a single or accelerated issue.

These terms are used for description of a new market of digital assets, namely cryptocurrencies, which has already gained a significant strength. This market is characterized by high volatility and it makes many investors to refer to this market with a great caution. Since the inception of the market in 2007 - 2010, crypto assets were considered as extremely risky investments. However, in the last few years the situation has been changing rapidly and more and more large investors include crypto assets in their portfolios. The following facts confirm these changes $[1,2]$ :

- Institutional investors begin to realize the potential of new technologies in the field of money, exchange, storage and validation of data, which were not available until the current day;

- Crypto assets are already not only a kind of fake for quick money earning, but an opportunity for many companies and startups to make ICO process easier and more transparent (the mentioned companies and startups have close relationships with the IT sector and blockchain technologies);

- Although this market is still very young nevertheless its consolidation is inevitable, so it will not disappear, and it becomes more evident;

- Crypto assets cost real money, they are backed by real technologies together with people who develop them

Effective functioning of cryptocurrencies market needs tools for the forecast of its state with satisfactory level of the confidence. It should say that the basic regularities of this market differ from the well-established markets such as Forex or securities markets. We proceed here from the following characteristics [3]:

- Entry threshold is much lower;

- Share of non-institutional investors is much higher;

- News background has a greater impact on player behavior;

- Cryptocurrency rate can be estimated by the description of the activities of companies - issuers.

These circumstances prove to be an obstacle for building confident models of cryptocurrencies value's middle-term forecast. So, speaking mid-term in this paper we mean both weekly and monthly forecasts.

\section{B. Related Work and Problem Setting}

This moment the unique reliable institution, which evaluates behavior of cryptocurrencies, is Weiss Agency. But its activity is limited only by ratings reflecting current state of various cryptocurrencies without any forecasts [4]. 
From the other hand Internet resources contain examples of forecast for different cryptocurrencies based on expert assessments. Practically all of them suggest not more than one-step forecast using qualitative scales and expert assessments $[5,6]$. In many cases the mentioned forecasts prove to be essentially different reflecting so-called information opposition in Internet [7].

Object of consideration in this paper is the cryptocurrency 'Waves'. Our interest to this cryptocurrency is explained by the following [8]:

- It is one of the youngest valuable cryptocurrency. It has been trading for the last 45 months (almost 4 years).

- It has the extremely high volatility. For example, during 2019 the volatility of Waves was equal up to $25 \%$ during one month

- The forecasts of this cryptocurrency are only one days with expert opinions as it is marked above [5]

In this paper we try to reduce the mentioned lacks. For this we build autoregressive models of forecast for Waves using GMDH Shell platform [9]. The following 2 circumstances justify such an approach:

1. Autoregression allows not taking into account external factors related to cryptocurrency rate. We suppose that these factors are hidden in the data themselves and these factors may manifest themselves in a long lags we use in modeling.

2. GMDH Shell contains several GMDH-based algorithms, which allows building models of optimal complexity under high uncertainty concerning its structure and parameters. Such models provide a balance between the accuracy of complex models and the noise immunity of simple models $[10,11]$.

Our contribution to the paper is study possibilities of different algorithms from GMDH Shell platform to provide weekly and monthly forecast of Waves rate and compare it with a well-known baseline. We consider the following cases:

- 3 year period of weakly registered data since January 2017 till December 2019, totally 156 weeks;

- $\quad 3$ quarters of weekly registered data during the crisis since October 2017 till June 2018, totally 39 weeks.

In both cases we calculate average accuracy both weekly and monthly forecasts on the last quarter. It is OctoberDecember 2019 for the 1-st case, and April-June 2018 for the 2-nd case. Accuracy of these forecasts is measured with NRMSE.

The quality of all the results is compared with the forecast of the traditional exponential smoothing with a linear trend. This model is also titled as double exponential smoothing [12]. Its advantages are well-known: simplicity of tuning and adaptability to data. The forecast with this popular model is considered as a Baseline.

\section{DATA AND TOOLS}

The source information is week data of waves/euro rate. Each value is averaged value of everyday data during a week. The data set covers 3 years; it is equal $52 \times 3=156$ weeks. The period of crisis lasted 3 quarters, it is equal $13 \mathrm{x}$ $3=39$ weeks. Table 1 describes statistical characteristics of 3 datasets. It is easy to see, that the variation on the period of crisis is 3 times more than that on the calm periods and 2 times more than that on the whole period. It is easy to see that the whole data set is some averaged case. Figures 1, 2 reflect dynamics of Waves during the whole period and during the crisis respectively.

TABLE I. CHARACTERISTICS OF DATASETS

\begin{tabular}{|l|c|c|c|}
\hline Parameter & $\begin{array}{c}\text { Full } \\
\text { period }\end{array}$ & $\begin{array}{c}\text { Without } \\
\text { crisis }\end{array}$ & Crisis \\
\hline Min & 0,20 & 0,20 & 2,71 \\
\hline Max & 14,72 & 5,68 & 14,72 \\
\hline Average & 3,12 & 2,05 & 6,32 \\
\hline Variation & 2,67 & 1,35 & 3,09 \\
\hline
\end{tabular}

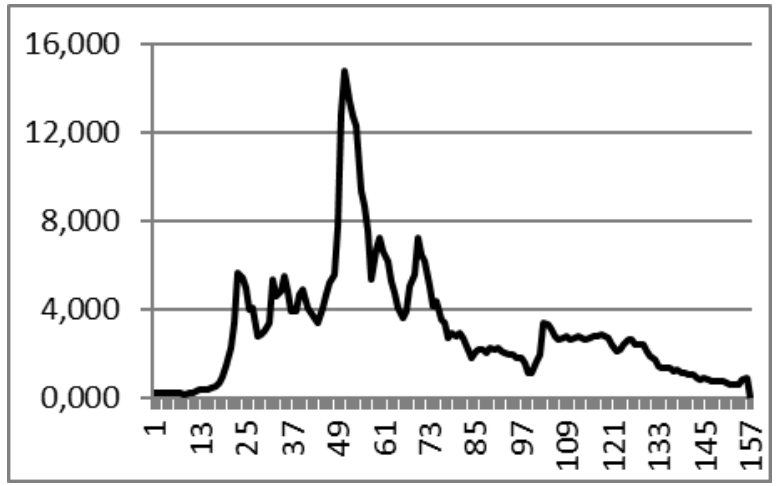

Fig. 1. Dynamics of Waves rate, full period (rate/days)



Fig. 2. Dynamics of Waves rate, period of crisis (rate/days)

\section{B. Group Method of Data Handling and GMDH Shell}

Group Method of Data Handling (GMDH) is a technology of machine learning (ML) for creating noise immunity models. The ideas and perspectives of GMDH are presented in many publications; see, for example, [10]. Theoretical bases of GMDH are described the most completely in [11]. Numerous applications of GMDH are reflected in papers and books, which can be downloaded from the resource [13].

\section{A. Dynamics of Data}


In our experimental research, we use algorithms from the famous platform GMDH Shell [9]. This platform offers the following 4 algorithms for forecast of time series:

1. Combi. It is a classical GMDH-based sort out algorithm, which considers all possible combinations of variables;

2. Neuro. It is also a GMDH-based neuro-similar relaxation algorithm, where generated variables are used together with the initial ones;

3. Forward. It is similar to the stepwise regression, where the procedure adds new member to a current model having tested it according principles of GMDH;

4. Mixed. It is similar to the stepwise regression, where the procedure may add successful members to a current model and also delete the unsuccessful ones from a current model having tested those according principles of GMDH.

A user has an opportunity to define and limit the class of polynomial models. For example, he/she can specify:

- Regression, autoregression, or hybrid model;

- Form of variables and the maximum number of members in a model (Combi, Forward, Mixed);

- Form of generative function and width of neuron layer (Neuro);

- Etc.

A user has possibility to use different criteria for assessment of the quality of forecast as training-testing, kfold cross validation, and also different measures of error. GMDH Shell before building a model analyses the given data and proposes the best options. A user can agree or not agree and make own choice.

\section{EXPERIMENTS WITH THE GMDH SHELL}

\section{A. Tuning}

To reach the best results the following options were proposed us by GMDH Shell and then manually corrected taking into account recommendations from [14]:

The model quality is evaluated with 2 -fold cross validation strategy. It is the same as the symmetric criterion of regularity [10]. The errors are measured by normalized root-mean-square error (NRMSE). Time lag equals 1 month (4 weeks) for weekly forecast and 3 months (12 weeks) for monthly forecast. Our experiments showed that smaller lags worsened result and bigger lags didn't affect it.

\section{B. Experiments}

The experiments aim to determine the averaged error of weekly and monthly forecast on the basis of the whole period and the period of crisis. The results of calculations are shown in Tables 2 and 3 for each algorithm including the Basic algorithm. The graphical illustration is presented on Figures 3 and 4.

\section{CONCLUSION}

\section{A. Results}

Subject of consideration in the paper are 4 algorithms from the platform GMDH Shell: Combi, Neuro, Forward, and Mixed. With these algorithms we build autoregressive model to forecast the dynamics of cryptocurrency Waves. Object of consideration are weekly data of Waves/USD rate during the period January 2017 - December 2019 (3 years). This dataset includes the period of crisis since October 2017 till June 2018 (3 quarters). We compare the quality of weekly and monthly forecasts with the baseline. The latter is the result of forecast by the method of double exponential smoothing (Holt-Winters method). To reach the best results we tune both the GMDH-based algorithms and the Basic algorithm.

TABLE II. NRMSE [\%], THE WHOLE PERIOD

\begin{tabular}{|c|c|c|}
\hline Algorithm & Weekly & Monthly \\
\hline Combi & 0,70 & 1,50 \\
\hline Neuro & 0,70 & 1,68 \\
\hline Forward & 0,69 & 1,52 \\
\hline Mixed & 0,69 & 1,26 \\
\hline Basic & 6,01 & 15,27 \\
\hline
\end{tabular}

TABLE III. NRMSE [\%], THE PERIOD OF CRISIS

\begin{tabular}{|l|c|c|}
\hline \multicolumn{1}{|c|}{ Algorithm } & Weekly & Monthly \\
\hline Combi & 10,07 & 20,23 \\
\hline Neuro & 9,85 & 19,85 \\
\hline Forward & 9,93 & 19,42 \\
\hline Mixed & 9,93 & 19,42 \\
\hline Basic & 22,17 & 61,40 \\
\hline
\end{tabular}

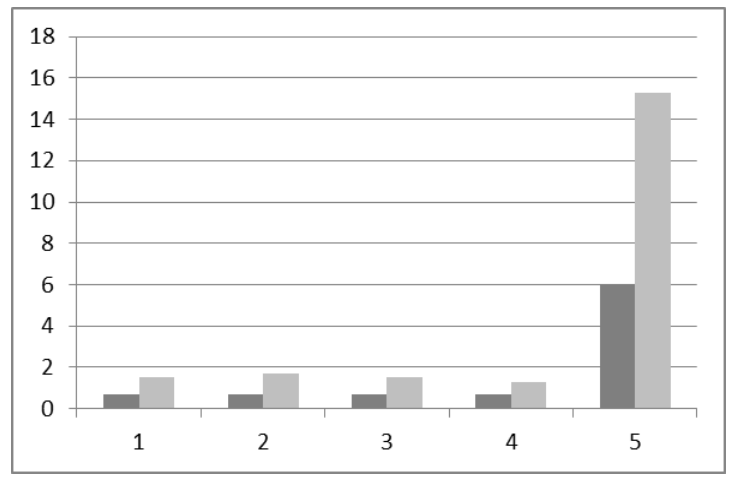

Fig. 3. Accuracy (NRSME) of weekly and monthly forecasts [\%] for the whole period: 1 - Combi, 2 - Neuro, 3 - Forward, 4 - Mixed, 5 - Basic 


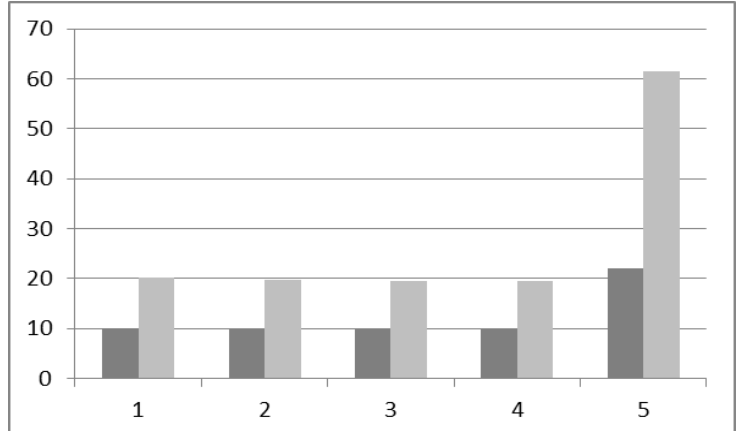

Fig. 4. Accuracy (NRSME) of weekly and monthly forecasts [\%] for the period of crisis: 1 - Combi, 2 - Neuro, 3 - Forward, 4 - Mixed, 5 - Basic

The results are the followings:

- All GMDH-based algorithms show very close results for each case: the whole period and the period of crisis, the weekly forecasts and the monthly forecasts. There are non-significant differences for monthly forecasts.

- For the whole period the results of GMDH-based algorithms for weekly forecasts are approximately 2 times better than that for monthly forecasts. The same we have for the period of crisis.

- For weekly forecasts the results of GMDH-based algorithms for the whole period are approximately 15 times better than that for the period of crisis. The same we have for monthly forecasts.

- For the whole period the results of GMDH-based algorithms are approximately 10 times better than that for the Basic algorithm. It refers both for weekly and for monthly forecasts

- For the crisis the results of GMDH-based algorithms are approximately 2 times and 3 times better than that for the Basic algorithm. It refers for weekly and for monthly forecasts respectively.

The results described above allow us to recommend GMDH-based algorithms as a perspective tool for forecasts of Waves/USD rate both for weakly and for monthly forecasts. Naturally, during the crisis this tool should be used with a large caution.

We would like to remind that the behaviour of cryptocurrencies is strongly depends on many subjective factors due to its nature. Our Pilot study demonstrates possibility of GMDH-based algorithms to provide a certain prediction of this behaviour under the absence of information concerning these factors.

\section{B. Future Work}

In future we suppose:
- To consider other cryptocurrencies, for example, XPR;

- To use qualitative scales of values;

- To consider joint forecast of cryptocurrencies having in view the effect of mutual contagion;

- To use additional sources of information, for example, expert opinions;

- To consider possibility of automatic switching between algorithms to take into account calm periods and periods of instability.

The latter proposal is similar to so-called intelligent modeling [15].

\section{REFERENCES}

[1] Cryptocurrency Market Capitalisations; https://coinmarketcap.com

[2] Analytical column; https:/u.today/guides/crypto-trading/every-rippleprice-prediction-2019-says-the-same-thing-xrp-price-might-reach-10

[3] P. Mogilev, "The state of the cryptocurrency market in 2018 and the expectations of governments and people in 2019 (review)“, In: Mathematical modelling of social processes, M: Keldysh Institute of Applied Mathematics, vol. 21, 2019; http://keldysh.ru/social/2019 [rus]

[4] M.D. Weiss, J.M. Villaverde, "Dark Shadows with a Bright Future," Weiss Crypto Report, March 2019 Coin Predictor Resource: Waves Price Prediction, https://coinpredictor.io/waves

[5] Coin Predictor Resource: Waves Price Prediction, https://coinpredictor.io/waves

[6] Coin Predictor Resource: Waves Price Check Up, https://coincheckup.com/coins/waves/

[7] Petrov, O. Proncheva, "Propaganda battle with two-component agenda," In: Proc. of the MACSPro (Workshop 2019), Austria, CEUR, vol. 478, 2019, pp. 28-38; http://ceur-ws.org/vol-2478/

[8] Waves blockchain technology; https://wavesplatform.com

[9] Platform GMDH Shell; http//www.gmdhshell.com

[10] V. Stepashko, "Developments and prospects of GMDH-based inductive modeling," Advances in Intelligent Systems and Computing II; Springer, AISC book series, vol. 689, 2017, pp. 346-360.

[11] V. Stepashko, "Method of critical variances as analytical tool of theory of inductive modeling," J. Autom. Inf. Sci., vol. 40, no. 3, 2008, pp. 4-22.

[12] C. Holt, "Forecasting trends and seasonals by exponentially weighted averages," Intern. J. of Forecasting, vol. 20, no. 1, 2004, pp. 5-10.

[13] GMDH Resource in IRTC ITS NAS of Ukraine; http//mgua.irtc.org.ua/

[14] O. Koshulko, G. Koshulko, "Validation strategy selection in combinatorial and multilayered iterative GMDH algorithms," Proc. 4th Intern. Workshop on Inductive Modeling (IWIM-2011), IRTC ITS of the NAS of Ukraine, Kyiv, 2011, pp. 51-54.

[15] V. Stepashko, "On the self-organizing inductive-based intelligent modeling," Advances in Intelligent Systems and Computing III. Springer, AISC book series, vol. 871, 2018, pp. 433-448; DOI: 10.1007/978-3-030-01069-0_31 